\title{
Inviscid limit for SQG in bounded domains
}

\author{
Peter Constantin, Mihaela Ignatova, and Huy Q. Nguyen
}

\begin{abstract}
We prove that the limit of any weakly convergent sequence of Leray-Hopf solutions of dissipative SQG equations is a weak solution of the inviscid SQG equation in bounded domains.
\end{abstract}

\section{Introduction}

The behavior of high Reynolds number fluids is a broad, important and mostly open problem of nonlinear physics and of PDE. Here we consider a model problem, the surface quasi-geostrophic equation, and the limit of its viscous regularizations of certain types. We prove that the inviscid limit is rigid, and no anomalies arise in the limit.

Let $\Omega \subset \mathbb{R}^{2}$ be a bounded domain with smooth boundary. Denote

$$
\Lambda=\sqrt{-\Delta}
$$

where $-\Delta$ is the Laplacian operator with Dirichlet boundary conditions. The dissipative surface quasigeostrophic (SQG) equation in $\Omega$ is the equation

$$
\partial_{t} \theta^{\nu}+u^{\nu} \cdot \nabla \theta^{\nu}+\nu \Lambda^{s} \theta^{\nu}=0, \quad \nu>0, s \in(0,2],
$$

where $\theta^{\nu}=\theta^{\nu}(x, t), u^{\nu}=u^{\nu}(x, t)$ with $(x, t) \in \Omega \times[0, \infty)$ and with the velocity $u^{\nu}$ given by

$$
u^{\nu}=R_{D}^{\perp} \theta^{\nu}:=\nabla^{\perp} \Lambda^{-1} \theta^{\nu}, \quad \nabla^{\perp}=\left(-\partial_{2}, \partial_{1}\right) .
$$

We refer to the parameter $\nu$ as "viscosity". Fractional powers of the Laplacian $-\Delta$ are based on eigenfunction expansions. The inviscid SQG equation has zero viscosity

$$
\partial_{t} \theta+u \cdot \nabla \theta=0, \quad u=R_{D}^{\perp} \theta .
$$

The dissipative SQG (1.1) has global weak solutions for any $L^{2}$ initial data:

THEOREM 1.1. For any initial data $\theta_{0} \in L^{2}(\Omega)$ there exists a global weak solution $\theta$

$$
\theta \in C_{w}\left(0, \infty ; L^{2}(\Omega)\right) \cap L^{2}\left(0, \infty ; D\left(\Lambda^{\frac{s}{2}}\right)\right)
$$

to the dissipative SQG equation (1.1). More precisely, $\theta$ satisfies the weak formulation

$$
\int_{0}^{\infty} \int_{\Omega} \theta \varphi(x) d x \partial_{t} \phi(t) d t+\int_{0}^{\infty} \int_{\Omega} u \theta \cdot \nabla \varphi(x) d x \phi(t) d t-\nu \int_{0}^{\infty} \int_{\Omega} \Lambda^{\frac{s}{2}} \theta \Lambda^{\frac{s}{2}} \varphi(x) d x \phi(t) d t=0
$$

for any $\phi \in C_{c}^{\infty}((0, \infty))$ and $\varphi \in D\left(\Lambda^{2}\right)$. Moreover, $\theta$ obeys the energy inequality

$$
\frac{1}{2}\|\theta(\cdot, t)\|_{L^{2}(\Omega)}^{2}+\nu \int_{0}^{t} \int_{\Omega}\left|\Lambda^{\frac{s}{2}} \theta\right|^{2} d x d r \leq \frac{1}{2}\left\|\theta_{0}\right\|_{L^{2}(\Omega)}^{2}
$$

and the balance

$$
\frac{1}{2}\|\theta(\cdot, t)\|_{D\left(\Lambda^{-\frac{1}{2}}\right)}^{2}+\nu \int_{0}^{t} \int_{\Omega}\left|\Lambda^{\frac{s-1}{2}} \theta\right|^{2} d x d r=\frac{1}{2}\left\|\theta_{0}\right\|_{D\left(\Lambda^{-\frac{1}{2}}\right)}^{2}
$$

for a.e. $t>0$. In addition, $\theta \in C\left([0, \infty) ; D\left(\Lambda^{-\varepsilon}\right)\right)$ for any $\varepsilon>0$ and the initial data $\theta_{0}$ is attained in $D\left(\Lambda^{-\varepsilon}\right)$. 
We refer to any weak solutions of (1.1) satisfying the properties (1.4), (1.5), (1.6) as a "Leray-Hopf weak solution".

REMARK 1.2. Theorem 1.1 for critical dissipative $S Q G s=1$ was obtained in [5].

REMARK 1.3. Note that $C_{c}^{\infty}(\Omega)$ is not dense in $D\left(\Lambda^{2}\right)$ since the $D\left(\Lambda^{2}\right)$ norm is equivalent to the $H^{2}(\Omega)$ norm and $C_{c}^{\infty}(\Omega)$ is dense in $H_{0}^{2}(\Omega)$ which is strictly contained in $D\left(\Lambda^{2}\right)$.

The existence of $L^{2}$ global weak solutions for inviscid SQG (1.3) was proved in [7]. More precisely, (see Theorem 1.1, [7]) for any initial data $\theta_{0} \in L^{2}(\Omega)$ there exists a global weak solution $\theta \in C_{w}\left(0, \infty ; L^{2}(\Omega)\right)$ satisfying

$$
\int_{0}^{\infty} \int_{\Omega} \theta \partial_{t} \varphi d x d t+\int_{0}^{\infty} \int_{\Omega} u \theta \cdot \nabla \varphi d x d t=0 \quad \forall \varphi \in C_{c}^{\infty}(\Omega \times(0, \infty)),
$$

and such that the Hamiltonian

$$
H(t):=\|\theta(t)\|_{D\left(\Lambda^{-\frac{1}{2}}\right)}^{2}
$$

is constant in time. Moreover, the initial data is attained in $D\left(\Lambda^{-\varepsilon}\right)$ for any $\varepsilon>0$.

Our main result in this note establishes the convergence of weak solutions of the dissipative SQG to weak solutions of the inviscid SQG in the inviscid limit $\nu \rightarrow 0$.

THEOREM 1.4. Let $\left\{\nu_{n}\right\}$ be a sequence of viscosities converging to 0 and let $\left\{\theta_{0}^{\nu_{n}}\right\}$ be a bounded sequence in $L^{2}(\Omega)$. Any weak limit $\theta$ in $L^{2}\left(0, T ; L^{2}(\Omega)\right), T>0$, of any subsequence of $\left\{\theta^{\nu_{n}}\right\}$ of Leray-Hopf weak solutions of the dissipative $S Q G$ equation (1.1) with viscosity $\nu_{n}$ and initial data $\theta_{0}^{\nu_{n}}$ is a weak solution of the inviscid SQG equation (1.3) on $[0, T]$. Moreover, $\theta \in C\left(0, T ; D\left(\Lambda^{-\varepsilon}\right)\right)$ for any $\varepsilon>0$, and when $S \in(0,1]$ the Hamiltonian of $\theta$ is constant on $[0, T]$.

REMARK 1.5. The same result holds true on the torus $\mathbb{T}^{2}$. The case of the whole space $\mathbb{R}^{2}$ was treated in [1].

REMARK 1.6. With more singular constitutive laws $u=\nabla^{\perp} \Lambda^{-\alpha} \theta, \alpha \in[0,1), L^{2}$ global weak solutions of the inviscid equations were obtained in $[3,14]$. Theorem 1.4 could be extended to this case. It is also possible to consider $L^{p}$ initial data in light of the work [11].

As a corollary of the proof of Theorem 1.4 we have the following weak rigidity of inviscid SQG in bounded domains:

COROLlARY 1.7. Any weak limit in $L^{2}\left(0, T ; L^{2}(\Omega)\right), T>0$, of any sequence of weak solutions of the inviscid SQG equation (1.3) is a weak solution of (1.3). Here, weak solutions of (1.3) are interpreted in the sense of (1.7).

REMARK 1.8. On tori, this result was proved in [13]. If the weak limit occurs in $L^{\infty}\left(0, T ; L^{2}(\Omega)\right)$ and the sequence of weak solutions conserves the Hamiltonian then so is the limiting weak solution.

The paper is organized as follows. Section 2 is devoted to basic facts about the spectral fractional Laplacian and results on commutator estimate. The proofs of Theorems 1.1 and 1.4 are given respectively in sections 3 and 4. Finally, an auxiliary lemma is given in Appendix A

\section{Fractional Laplacian and commutators}

Let $\Omega \subset \mathbb{R}^{d}, d \geq 2$, be a bounded domain with smooth boundary. The Laplacian $-\Delta$ is defined on $D(-\Delta)=H^{2}(\Omega) \cap H_{0}^{1}(\Omega)$. Let $\left\{w_{j}\right\}_{j=1}^{\infty}$ be an orthonormal basis of $L^{2}(\Omega)$ comprised of $L^{2}$-normalized eigenfunctions $w_{j}$ of $-\Delta$, i.e.

$$
-\Delta w_{j}=\lambda_{j} w_{j}, \quad \int_{\Omega} w_{j}^{2} d x=1,
$$


with $0<\lambda_{1}<\lambda_{2} \leq \ldots \leq \lambda_{j} \rightarrow \infty$.

The fractional Laplacian is defined using eigenfunction expansions,

$$
\Lambda^{s} f \equiv(-\Delta)^{\frac{s}{2}} f:=\sum_{j=1}^{\infty} \lambda_{j}^{\frac{s}{2}} f_{j} w_{j} \quad \text { with } f=\sum_{j=1}^{\infty} f_{j} w_{j}, \quad f_{j}=\int_{\Omega} f w_{j} d x
$$

for $s \geq 0$ and $f \in D\left(\Lambda^{s}\right):=\left\{f \in L^{2}(\Omega):\left(\lambda_{j}^{\frac{s}{2}} f_{j}\right) \in \ell^{2}(\mathbb{N})\right\}$. The norm of $f$ in $D\left(\Lambda^{s}\right)$ is defined by

$$
\|f\|_{D\left(\Lambda^{s}\right)}:=\left\|\left(\lambda_{j}^{\frac{s}{2}} f_{j}\right)\right\|_{\ell^{2}(\mathbb{N})} .
$$

It is also well-known that $D(\Lambda)$ and $H_{0}^{1}(\Omega)$ are isometric. In the language of interpolation theory,

$$
D\left(\Lambda^{\alpha}\right)=\left[L^{2}(\Omega), D(-\Delta)\right]_{\frac{\alpha}{2}} \quad \forall \alpha \in[0,2] .
$$

As mentioned above,

$$
H_{0}^{1}(\Omega)=D(\Lambda)=\left[L^{2}(\Omega), D(-\Delta)\right]_{\frac{1}{2}}
$$

hence

$$
D\left(\Lambda^{\alpha}\right)=\left[L^{2}(\Omega), H_{0}^{1}(\Omega)\right]_{\alpha} \quad \forall \alpha \in[0,1] .
$$

Consequently, we can identify $D\left(\Lambda^{\alpha}\right)$ with usual Sobolev spaces (see Chapter 1, [16]):

$$
D\left(\Lambda^{\alpha}\right)= \begin{cases}H_{0}^{\alpha}(\Omega) & \text { if } \alpha \in\left(\frac{1}{2}, 1\right], \\ H_{00}^{\frac{1}{2}}(\Omega):=\left\{u \in H_{0}^{\frac{1}{2}}(\Omega): u / \sqrt{d(x)} \in L^{2}(\Omega)\right\} & \text { if } \alpha=\frac{1}{2}, \\ H^{\alpha}(\Omega) & \text { if } \alpha \in\left[0, \frac{1}{2}\right) .\end{cases}
$$

Here and below $d(x)$ denote the distance from $x$ to the boundary $\partial \Omega$.

Next, for $s>0$ we define

$$
\Lambda^{-s} f=\sum_{j=1}^{\infty} \lambda_{j}^{-\frac{s}{2}} f_{j} w_{j}
$$

if $f=\sum_{j=1}^{\infty} f_{j} w_{j} \in D\left(\Lambda^{-s}\right)$ where

$$
D\left(\Lambda^{-s}\right):=\left\{\sum_{j=1}^{\infty} f_{j} w_{j} \in \mathscr{D}^{\prime}(\Omega): f_{j} \in \mathbb{R}, \sum_{j=1}^{\infty} \lambda_{j}^{-\frac{s}{2}} f_{j} w_{j} \in L^{2}(\Omega)\right\} .
$$

The norm of $f$ is then defined by

$$
\|f\|_{D\left(\Lambda^{-s}\right)}:=\left\|\Lambda^{-s} f\right\|_{L^{2}(\Omega)}=\left(\sum_{j=1}^{\infty} \lambda_{j}^{-s} f_{j}^{2}\right)^{\frac{1}{2}} .
$$

It is easy to check that $D\left(\Lambda^{-S}\right)$ is the dual of $D\left(\Lambda^{s}\right)$ with respect to the pivot space $L^{2}(\Omega)$.

LEMMA 2.1 (Lemma 2.1, [14]). The embedding

$$
D\left(\Lambda^{S}\right) \subset H^{S}(\Omega)
$$

is continuous for all $s \geq 0$.

Lemma 2.2. For $s, r \in \mathbb{R}$ with $s>r$, the embedding $D\left(\Lambda^{s}\right) \subset D\left(\Lambda^{r}\right)$ is compact.

Proof. Let $\left\{u_{n}\right\}$ be a bounded sequence in $D\left(\Lambda^{s}\right)$. Then $\left\{\Lambda^{r} u_{n}\right\}$ is bounded in $D\left(\Lambda^{s-r}\right)$. Choosing $\delta>0$ smaller than $\min \left(s-r, \frac{1}{2}\right)$ we have $D\left(\Lambda^{s-r}\right) \subset D\left(\Lambda^{\delta}\right)=H^{\delta}(\Omega) \subset L^{2}(\Omega)$ where the first embedding is continuous and the second is compact. Consequently the embedding $D\left(\Lambda^{s-r}\right) \subset L^{2}(\Omega)$ is compact and thus there exist a subsequence $n_{j}$ and a function $f \in L^{2}(\Omega)$ such that $\Lambda^{r} u_{n_{j}}$ converge to $f$ strongly in $L^{2}(\Omega)$. Then $u_{n_{j}}$ converge to $u:=\Lambda^{-r} f$ strongly in $D\left(\Lambda^{r}\right)$ and the proof is complete. 
A bound for the commutator between $\Lambda$ and multiplication by a smooth function was proved in [5] using the method of harmonic extension:

THEOREM 2.3 (Theorem 2, [5] ). Let $\chi \in B(\Omega)$ with $B(\Omega)=W^{2, d}(\Omega) \cap W^{1, \infty}(\Omega)$ if $d \geq 3$, and $B(\Omega)=$ $W^{2, p}(\Omega)$ with $p>2$ if $d=2$. There exists a constant $C(d, p, \Omega)$ such that

$$
\|[\Lambda, \chi] \psi\|_{D\left(\Lambda^{\frac{1}{2}}\right)} \leq C(d, p, \Omega)\|\chi\|_{B(\Omega)}\|\psi\|_{D\left(\Lambda^{\frac{1}{2}}\right)} .
$$

Pointwise estimates for the commutator between fractional Laplacian and differentiation were established in [7]:

THEOREM 2.4 (Theorem 2.2, [7]). For any $p \in[1, \infty]$ and $s \in(0,2)$ there exists a positive constant $C(d, s, p, \Omega)$ such that for all $\psi \in C_{c}^{\infty}(\Omega)$ we have

$$
\left|\left[\Lambda^{s}, \nabla\right] \psi(x)\right| \leq C(d, s, p, \Omega) d(x)^{-s-1-\frac{d}{p}}\|\psi\|_{L^{p}(\Omega)}
$$

holds for all $x \in \Omega$.

This pointwise bound implies the following commutator estimate in Lebesgue spaces.

THEOREM 2.5. Let $p, q \in[1, \infty], s \in(0,2)$ and $\varphi$ satisfy

$$
\varphi(\cdot) d(\cdot)^{-s-1-\frac{d}{p}} \in L^{q}(\Omega) .
$$

Then the operator $\varphi\left[\Lambda^{s}, \nabla\right]$ can be uniquely extended from $C_{c}^{\infty}(\Omega)$ to $L^{p}(\Omega)$ such that there exists a positive constant $C=C(d, s, p, \Omega)$ such that

$$
\left\|\varphi\left[\Lambda^{s}, \nabla\right] \psi\right\|_{L^{q}(\Omega)} \leq C\left\|\varphi(\cdot) d(\cdot)^{-S-1-\frac{d}{p}}\right\|_{L^{q}(\Omega)}\|\psi\|_{L^{p}(\Omega)}
$$

holds for all $\psi \in L^{p}(\Omega)$.

The inequality (2.3) is remarkable because the commutator between an operator of order $s \in(0,2)$ and an operator of order 1 is an operator of order 0 .

\section{Proof of Theorem 1.1}

We use Galarkin approximations. Denote by $\mathbb{P}_{m}$ the projection in $L^{2}(\Omega)$ onto the linear span $L_{m}^{2}$ of eigenfunctions $\left\{w_{1}, \ldots, w_{m}\right\}$, i.e.

$$
\mathbb{P}_{m} f=\sum_{j=1}^{m} f_{j} w_{j} \quad \text { for } f=\sum_{j=1}^{\infty} f_{j} w_{j} .
$$

The $m$ th Galerkin approximation of 1.1 is the following ODE system in the finite dimensional space $L_{m}^{2}$ :

$$
\begin{cases}\dot{\theta}_{m}+\mathbb{P}_{m}\left(u_{m} \cdot \nabla \theta_{m}\right)+\nu \Lambda^{s} \theta_{m}=0 & t>0 \\ \theta_{m}=P_{m} \theta_{0} & t=0\end{cases}
$$

with $\theta_{m}(x, t)=\sum_{j=1}^{m} \theta_{j}^{(m)}(t) w_{j}(x)$ and $u_{m}=R_{D}{ }^{\perp} \theta_{m}$ satisfying $\operatorname{div} u_{m}=0$. Note that (3.2) is equivalent to

$$
\frac{d \theta_{l}^{(m)}}{d t}+\sum_{j, k=1}^{m} \gamma_{j k l}^{(m)} \theta_{j}^{(m)} \theta_{k}^{(m)}+\nu \lambda_{l}^{\frac{s}{2}} \theta_{l}^{(m)}=0, \quad l=1,2, \ldots, m,
$$

with

$$
\gamma_{j k l}^{(m)}=\lambda_{j}^{-\frac{1}{2}} \int_{\Omega}\left(\nabla^{\perp} w_{j} \cdot \nabla w_{k}\right) w_{l} d x
$$


The local existence of $\theta_{m}$ on some time interval $\left[0, T_{m}\right]$ follows from the Cauchy-Lipschitz theorem. On the other hand, the antisymmetry property $\gamma_{j k l}^{(m)}=-\gamma_{j l k}^{(m)}$ yields

$$
\frac{1}{2}\left\|\theta_{m}(\cdot, t)\right\|_{L^{2}(\Omega)}^{2}+\nu \int_{0}^{t} \int_{\Omega}\left|\Lambda^{\frac{s}{2}} \theta_{m}\right|^{2} d x d r=\frac{1}{2}\left\|\mathbb{P}_{m} \theta_{0}\right\|_{L^{2}(\Omega)}^{2} \leq \frac{1}{2}\left\|\theta_{0}\right\|_{L^{2}(\Omega)}^{2}
$$

for all $t \in\left[0, T_{m}\right]$. This implies that $\theta_{m}$ is global and (3.4) holds for all positive times. The sequence $\theta_{m}$ is thus uniformly bounded in $L^{\infty}\left(0, \infty ; L^{2}(\Omega)\right) \cap L^{2}\left(0, \infty ; D\left(\Lambda^{\frac{s}{2}}\right)\right)$. Upon extracting a subsequence, we have $\theta_{m}$ converge to some $\theta$ weakly-* in $L^{\infty}\left(0, \infty ; L^{2}(\Omega)\right)$ and weakly in $L^{2}\left(0, \infty ; D\left(\Lambda^{\frac{s}{2}}\right)\right)$. In particular, $\theta$ obeys the same energy inequality as in (3.4). On the other hand, if one multiplies (3.3) by $\lambda_{l}^{-1 / 2} \theta_{l}^{(m)}$ and uses the fact that $\gamma_{j k l}^{(m)} \lambda_{l}^{-1 / 2}=-\gamma_{l k j}^{(m)} \lambda_{j}^{-1 / 2}$, one obtains

$$
\frac{1}{2}\left\|\theta_{m}(\cdot, t)\right\|_{D\left(\Lambda^{-\frac{1}{2}}\right)}^{2}+\nu \int_{0}^{t} \int_{\Omega}\left|\Lambda^{\frac{s-1}{2}} \theta_{m}\right|^{2} d x d r=\frac{1}{2}\left\|\mathbb{P}_{m} \theta_{0}\right\|_{D\left(\Lambda^{-\frac{1}{2}}\right)}^{2} .
$$

We derive next a uniform bound for $\partial_{t} \theta_{m}$. Let $N>0$ be an integer to be determined. For any $\varphi \in D\left(\Lambda^{2 N}\right)$ we integrate by parts to get

$$
\begin{aligned}
\int_{\Omega} \partial_{t} \theta_{m} \varphi d x=- & \int_{\Omega} \mathbb{P}_{m} \operatorname{div}\left(u_{m} \theta_{m}\right) \varphi d x-\int_{\Omega} \nu \Lambda^{s} \theta_{m} \varphi d x \\
& =\int_{\Omega}\left(u_{m} \theta_{m}\right) \cdot \nabla\left(\mathbb{P}_{m} \varphi\right) d x-\int_{\Omega} \nu \theta_{m} \Lambda^{s} \phi d x .
\end{aligned}
$$

The first term is controlled by

$$
\left|\int_{\Omega}\left(u_{m} \theta_{m}\right) \cdot \nabla\left(\mathbb{P}_{m} \varphi\right) d x\right| \leq\left\|u_{m} \theta_{m}\right\|_{L^{1}(\Omega)}\left\|\nabla \mathbb{P}_{m} \varphi\right\|_{L^{\infty}(\Omega)} \leq C\left\|\mathbb{P}_{m} \varphi\right\|_{H^{3}(\Omega)} .
$$

According to Lemma A.1, for $N$ and $k$ satisfying $N>\frac{k}{2}+1$ there exists a positive constant $C_{N, k}$ such that

$$
\left\|\mathbb{P}_{m} \varphi\right\|_{H^{k}(\Omega)} \leq C_{N, k}\|\varphi\|_{D\left(\Lambda^{2 N}\right)} \quad \forall m \geq 1, \forall \varphi \in D\left(\Lambda^{2 N}\right) .
$$

With $k=3$ and $N=3$ we have

$$
\left|\int_{\Omega}\left(u_{m} \theta_{m}\right) \cdot \nabla\left(\mathbb{P}_{m} \varphi\right) d x\right| \leq C\|\varphi\|_{D\left(\Lambda^{6}\right)}
$$

On the other hand,

$$
\left|\int_{\Omega} \nu \theta_{m} \Lambda^{\mathrm{s}} \varphi d x\right| \leq C\left\|\theta_{m}\right\|_{L^{2}(\Omega)}\|\varphi\|_{D\left(\Lambda^{2}\right)}
$$

We have proved that

$$
\left|\int_{\Omega} \partial_{t} \theta_{m} \varphi d x\right| \leq C\|\varphi\|_{D\left(\Lambda^{6}\right)} \quad \forall \varphi \in D\left(\Lambda^{6}\right)
$$

Because $L^{2}(\Omega) \times D\left(\Lambda^{6}\right) \ni(f, g) \mapsto \int_{\Omega} f g d x$ extends uniquely to a bilinear from on $D\left(\Lambda^{-6}\right) \times D\left(\Lambda^{6}\right)$, we deduce that $\partial_{t} \theta_{m}$ are uniformly bounded in $L^{\infty}\left(0, \infty ; D\left(\Lambda^{-6}\right)\right)$. Note that we have used only the uniform regularity $L^{\infty}\left(0 ; \infty ; L^{2}(\Omega)\right)$ of $\theta_{m}$. We have the embeddings $D\left(\Lambda^{\frac{s}{2}}\right) \subset D\left(\Lambda^{(s-1) / 2}\right) \subset D\left(\Lambda^{-6}\right)$ where the first one is compact by virtue of Lemma 2.2, and the second is continuous. Fix $T>0$. AubinLions' lemma (see [15]) ensures that for some function $f$ and along some subsequence $\theta_{m}$ converge to $f$ weakly in $L^{2}\left(0, T ; D\left(\Lambda^{\frac{s}{2}}\right)\right)$ and strongly in $L^{2}\left(0, T ; D\left(\Lambda^{(s-1) / 2}\right)\right)$. In principle, both $f$ and the subsequence might depend on $T$, however, we already know that $\theta_{m} \rightarrow \theta$ weakly in $L^{2}\left(0, \infty ; D\left(\Lambda^{\frac{s}{2}}\right)\right)$. Therefore, $f=\theta$ and the convergences to $\theta$ hold for the whole sequence. Similarly, applying Aubin-Lions' lemma with the embeddings $L^{2}(\Omega) \subset D\left(\Lambda^{-\varepsilon}\right) \subset D\left(\Lambda^{-6}\right)$ for sufficiently small $\varepsilon>0$ we obtain that $\theta_{m} \rightarrow \theta$ 
strongly in $C\left([0, T] ; D\left(\Lambda^{-\varepsilon}\right)\right)$. Integrating (3.2) against an arbitrary test function of the form $\phi(t) \varphi(x)$ with $\phi \in C_{c}^{\infty}((0, T)), \varphi \in D\left(\Lambda^{6}\right)$ yields

$\int_{0}^{T} \int_{\Omega} \theta_{m} \varphi(x) d x \partial_{t} \phi(t) d t+\int_{0}^{T} \int_{\Omega} u_{m} \theta_{m} \cdot \nabla \mathbb{P}_{m} \varphi(x) d x \phi(t) d t-\nu \int_{0}^{T} \int_{\Omega} \Lambda^{\frac{s}{2}} \theta_{m} \Lambda^{\frac{s}{2}} \varphi(x) d x \phi(t) d t=0$.

By Lemma A.1,

$$
\left\|\left(\mathbb{I}-\mathbb{P}_{m}\right) \varphi\right\|_{L^{\infty}(\Omega)} \leq C\left\|\left(\mathbb{I}-\mathbb{P}_{m}\right) \varphi\right\|_{H^{3}(\Omega)} \rightarrow 0 \quad \text { as } m \rightarrow \infty .
$$

The weak convergence of $\theta_{m}$ in $L^{2}\left(0, T ; D\left(\Lambda^{\frac{s}{2}}\right)\right)$ allows one to pass to the limit in the two linear terms. The strong convergence of $\theta_{m}$ in $L^{2}\left(0, T ; L^{2}(\Omega)\right)$ together with the weak convergence of $u_{m}$ in the same space allows one to pass to the limit in the nonlinear term and conclude that $\theta$ satisfies the weak formulation (1.4) with $\varphi \in D\left(\Lambda^{6}\right)$. In fact, $\theta \in L^{2}\left(0, \infty ; D\left(\Lambda^{\frac{s}{2}}\right)\right) \subset L^{2}\left(0, \infty ; L^{p}(\Omega)\right)$ for some $p>2$, hence $u \theta \in$ $L^{2}\left(0, \infty ; L^{q}(\Omega)\right)$ for some $q>1$. In addition, if $\varphi \in D\left(\Lambda^{2}\right)$ then $\nabla \varphi \in L^{r}$ for all $r<\infty$, and thus the nonlinearity $\int_{\Omega} u \theta \cdot \nabla \varphi d x$ makes sense. Then because $D\left(\Lambda^{2}\right)$ is dense in $D\left(\Lambda^{6}\right)$, (1.4) holds for $\varphi \in D\left(\Lambda^{2}\right)$.

We now pass to the limit in (3.5). The strong convergence $\theta_{m} \rightarrow \theta$ in $C\left(0, T ; D\left(\Lambda^{-\varepsilon}\right)\right)$ gives the convergence of the first term. On the other hand, the strong convergence $\theta_{m} \rightarrow \theta$ in $L^{2}\left(0, T ; D\left(\Lambda^{(s-1) / 2}\right)\right)$ yields the convergence of the second term. The right hand side converges to $\frac{1}{2}\left\|\theta_{0}\right\|_{D\left(\Lambda^{-\frac{1}{2}}\right)}^{2}$ since $\mathbb{P}_{m} \theta_{0}$ converge to $\theta_{0}$ in $L^{2}(\Omega)$. We thus obtain (1.6).

Since $\theta_{m} \rightarrow \theta$ in $C\left([0, T] ; D\left(\Lambda^{-\varepsilon}\right)\right)$ we deduce that

$$
\theta_{0}=\lim _{m \rightarrow \infty} \mathbb{P}_{m} \theta_{0}=\left.\lim _{m \rightarrow \infty} \theta_{m}\right|_{t=0}=\left.\theta\right|_{t=0} \quad \text { in } D\left(\Lambda^{-\varepsilon}\right) .
$$

For a.e. $t \in[0, T], \theta_{m}(t)$ are uniformly bounded in $L^{2}(\Omega)$, and thus along some subsequence $m_{j}$, a priori depending on $t$, we have $\theta_{m_{j}}(t)$ converge weakly to some $f(t)$ in $L^{2}(\Omega)$. But we know $\theta_{m}(t) \rightarrow \theta(t)$ in $D\left(\Lambda^{-\varepsilon}\right)$. Thus, $f(t)=\theta(t)$ and $\theta_{m}(t) \rightarrow \theta(t)$ in $L^{2}(\Omega)$ as a whole sequence for a.e. $t \in[0, T]$. Recall that $\frac{d}{d t} \theta_{m}$ are uniformly bounded in $L^{\infty}\left(0, T ; D\left(\Lambda^{-6}\right)\right)$. For all $\varphi \in D\left(\Lambda^{6}\right)$ and $t \in[0, T]$ we write

$$
\left\langle\theta_{m}(t), \varphi\right\rangle_{L^{2}(\Omega), L^{2}(\Omega)}=\left\langle\theta_{m}(0), \varphi\right\rangle_{L^{2}(\Omega), L^{2}(\Omega)}+\int_{0}^{t}\left\langle\frac{d}{d t} \theta_{m}(r), \varphi\right\rangle_{D\left(\Lambda^{-6}\right), D\left(\Lambda^{6}\right)} d r .
$$

Because $\frac{d}{d t} \theta_{m}$ converge to $\frac{d}{d t} \theta$ weakly-* in $L^{\infty}\left(0, T ; D\left(\Lambda^{-6}\right)\right)$, letting $m \rightarrow \infty$ yields

$$
\langle\theta(t), \varphi\rangle_{L^{2}(\Omega), L^{2}(\Omega)}=\left\langle\theta_{0}, \varphi\right\rangle_{L^{2}(\Omega), L^{2}(\Omega)}+\int_{0}^{t}\left\langle\frac{d}{d t} \theta(r), \varphi\right\rangle_{D\left(\Lambda^{-6}\right), D\left(\Lambda^{6}\right)} d r
$$

for a.e. $t \in[0, T]$. Taking the limit $t \rightarrow 0$ gives

$$
\lim _{t \rightarrow 0}\langle\theta(t), \varphi\rangle_{L^{2}(\Omega), L^{2}(\Omega)}=\left\langle\theta_{0}, \varphi\right\rangle_{L^{2}(\Omega), L^{2}(\Omega)}
$$

for all $\varphi \in D\left(\Lambda^{6}\right)$. Finally, since $D\left(\Lambda^{6}\right)$ is dense in $L^{2}(\Omega)$ and $\theta \in L^{\infty}\left(0, T ; L^{2}(\Omega)\right)$ we conclude that $\theta \in C_{w}\left(0, T ; L^{2}(\Omega)\right)$ for all $T>0$.

\section{Proof of Theorem 1.4}

First, using approximations and commutator estimates we justify the commutator structure of the SQG nonlinearity derived in [7].

LEMmA 4.1. For all $\psi \in H_{0}^{1}(\Omega)$ and $\varphi \in C_{c}^{\infty}(\Omega)$ we have

$$
\int_{\Omega} \Lambda \psi \nabla^{\perp} \psi \cdot \nabla \varphi d x=\frac{1}{2} \int_{\Omega}\left[\Lambda, \nabla^{\perp}\right] \psi \cdot \nabla \varphi \psi d x-\frac{1}{2} \int_{\Omega} \nabla^{\perp} \psi \cdot[\Lambda, \nabla \varphi] \psi d x .
$$

Here, the commutator $\left[\Lambda, \nabla^{\perp}\right] \psi \cdot \nabla \varphi$ is understood in the sense of the extended operator defined in Theorem 2.5 
Proof. Let $\psi_{n} \in C_{c}^{\infty}(\Omega)$ converging to $\psi$ in $H_{0}^{1}(\Omega)$. Integrating by parts and using the fact that $\nabla^{\perp} \cdot \nabla \varphi=0$ gives

$$
\int_{\Omega} \Lambda \psi_{n} \nabla^{\perp} \psi_{n} \cdot \nabla \varphi d x=-\int_{\Omega} \psi_{n} \nabla^{\perp} \Lambda \psi_{n} \cdot \nabla \varphi d x
$$

Because $\psi_{n}$ is smooth and has compact support, $\nabla^{\perp} \psi_{n} \in D(\Lambda)$, and thus we can commute $\nabla^{\perp}$ with $\Lambda$ to obtain

$$
\begin{aligned}
& \int_{\Omega} \Lambda \psi_{n} \nabla^{\perp} \psi_{n} \cdot \nabla \varphi d x \\
& =-\int_{\Omega} \psi_{n}\left[\nabla^{\perp}, \Lambda\right] \psi_{n} \cdot \nabla \varphi d x-\int_{\Omega} \psi_{n} \Lambda \nabla^{\perp} \psi_{n} \cdot \nabla \varphi d x \\
& =-\int_{\Omega} \psi_{n}\left[\nabla^{\perp}, \Lambda\right] \psi_{n} \cdot \nabla \varphi d x-\int_{\Omega} \nabla^{\perp} \psi_{n} \cdot \Lambda\left(\psi_{n} \nabla \varphi\right) d x \\
& =-\int_{\Omega}\left[\nabla^{\perp}, \Lambda\right] \psi_{n} \cdot \nabla \varphi \psi_{n} d x-\int_{\Omega} \nabla^{\perp} \psi_{n} \cdot[\Lambda, \nabla \varphi] \psi_{n} d x-\int_{\Omega} \nabla^{\perp} \psi_{n} \cdot \nabla \varphi \Lambda \psi_{n} d x .
\end{aligned}
$$

Noticing that the last term on the right-hand side is exactly the negative of the left-hand side, we deduce that

$$
\int_{\Omega} \Lambda \psi_{n} \nabla^{\perp} \psi_{n} \cdot \nabla \varphi d x=\frac{1}{2} \int_{\Omega}\left[\Lambda, \nabla^{\perp}\right] \psi_{n} \cdot \nabla \varphi \psi_{n} d x-\frac{1}{2} \int_{\Omega} \nabla^{\perp} \psi_{n} \cdot[\Lambda, \nabla \varphi] \psi_{n} d x .
$$

The commutator estimates in Theorems 2.3 and 2.5 then allow us to pass to the limit in the preceding representation and conclude that 4.10 holds.

Now let $\nu_{n} \rightarrow 0^{+}$and let $\theta_{0}^{\nu_{n}}$ be a bounded sequence in $L^{2}(\Omega)$. For each $n$ let $\theta_{n} \equiv \theta^{\nu_{n}}$ be a Leray-Hopf weak solution of (1.1) with viscosity $\nu_{n}$ and initial data $\theta_{0}^{\nu_{n}}$. In view of the energy inequality (1.5), $\theta_{n}$ are uniformly bounded in $L^{\infty}\left(0, \infty ; L^{2}(\Omega)\right)$ and satisfies

$$
\int_{0}^{\infty} \int_{\Omega} \theta_{n} \varphi(x) d x \partial_{t} \phi(t) d t+\int_{0}^{\infty} \int_{\Omega} u_{n} \theta_{n} \cdot \nabla \varphi(x) d x \phi(t) d t-\nu_{n} \int_{0}^{\infty} \int_{\Omega} \Lambda^{\frac{s}{2}} \theta_{n} \Lambda^{\frac{s}{2}} \varphi(x) d x \phi(t) d t=0
$$

for all $\phi \in C_{c}^{\infty}((0, \infty))$ and $\varphi \in D\left(\Lambda^{2}\right)$. Fix $T>0$. Assume that along a subsequence, still labeled by $n, \theta_{n}$ converge to $\theta$ weakly in $L^{2}\left(0, T ; L^{2}(\Omega)\right)$. We prove that $\theta$ is a weak solution of the inviscid SQG equation. We first prove a uniform bound for $\partial_{t} \theta_{n}$ provided only the uniform regularity $L^{\infty}\left(0, T ; L^{2}(\Omega)\right)$ of $\theta_{n}$. To this end, let us define for a.e. $t \in[0, T]$ the function $f_{n}(\cdot, t) \in H^{-3}(\Omega)$ by

$$
\left\langle f_{n}(t), \varphi\right\rangle_{H^{-3}(\Omega), H_{0}^{3}(\Omega)}:=\int_{\Omega}\left(u_{n}(x, t) \theta_{n}(x, t) \cdot \nabla \varphi(x)-\nu_{n} \theta_{n}(x, t) \Lambda^{s} \varphi(x)\right) d x
$$

for all $\varphi \in H_{0}^{3}(\Omega) \subset D\left(\Lambda^{2}\right)$. Indeed, we have

$$
\left|\int_{\Omega}\left(u_{n}(x, t) \theta_{n}(x, t) \cdot \nabla \varphi(x)-\nu_{n} \theta_{n}(x, t) \Lambda^{s} \varphi(x)\right) d x\right| \leq C\left(\left\|\theta_{n}(t)\right\|_{L^{2}(\Omega)}^{2}+1\right)\|\varphi\|_{H^{3}(\Omega)} .
$$

This shows that $f_{n}$ are uniformly bounded in $L^{\infty}\left(0, T ; H^{-3}(\Omega)\right)$. Then for any $\phi \in C_{c}^{\infty}((0, T))$, it follows from (4.2) that

$$
\int_{0}^{T} \theta_{n} \partial_{t} \phi d t=-\int_{0}^{T} f_{n} \phi d t .
$$

In other words, $\partial_{t} \theta_{n}=f_{n}$ and the desired uniform bound for $\partial_{t} \theta_{n}$ follows. Fix $\varepsilon \in\left(0, \frac{1}{2}\right)$. Aubin-Lions' lemma applied with the embeddings $L^{2}(\Omega) \subset D\left(\Lambda^{-\varepsilon}\right) \subset H^{-3}(\Omega)$ then ensures that $\theta_{n}$ converge to $\theta$ strongly in $C\left(0, T ; D\left(\Lambda^{-\varepsilon}\right)\right) \subset C\left(0, T ; H^{-1}(\Omega)\right)$. Consequently $\psi_{n}$ converge to $\psi:=\Lambda^{-1} \theta$ strongly in $C\left(0, T ; L^{2}(\Omega)\right)$. 
Now we take $\phi \in C_{c}^{\infty}((0, \infty))$ and $\varphi \in C_{c}^{\infty}(\Omega)$. By virtue of Lemma4.1, the weak formulation (1.4) gives

$$
\begin{aligned}
\int_{0}^{T} & \int_{\Omega} \theta_{n} \varphi(x) d x \partial_{t} \phi(t) d t+\frac{1}{2} \int_{0}^{T} \int_{\Omega}\left[\Lambda, \nabla^{\perp}\right] \psi_{n} \cdot \nabla \varphi(x) \psi_{n} d x \phi(t) d t \\
& -\frac{1}{2} \int_{0}^{T} \int_{\Omega} \nabla^{\perp} \psi_{n} \cdot[\Lambda, \nabla \varphi(x)] \psi_{n} d x \phi(t) d t-\nu_{n} \int_{0}^{T} \int_{\Omega} \theta_{n} \Lambda^{s} \varphi(x) d x \phi(t) d t=0,
\end{aligned}
$$

where $\psi_{n}:=\Lambda^{-1} \theta_{n}$ are uniformly bounded in $L^{\infty}\left(0, T ; H_{0}^{1}(\Omega)\right)$. The weak convergence $\theta_{n} \rightarrow \theta$ in $L^{2}\left(0, T ; L^{2}(\Omega)\right)$ readily yields

$$
\lim _{n \rightarrow \infty} \int_{0}^{T} \int_{\Omega} \theta_{n} \varphi(x) d x \partial_{t} \phi(t) d t=\int_{0}^{T} \int_{\Omega} \theta \varphi(x) d x \partial_{t} \phi(t) d t
$$

and

$$
\lim _{n \rightarrow \infty} \nu_{n} \int_{0}^{T} \int_{\Omega} \theta_{n} \Lambda^{s} \varphi(x) d x \phi(t) d t=0
$$

Next we pass to the limit in the two nonlinear terms. Applying the commutator estimate in Theorem 2.3 we have

$$
\begin{aligned}
& \left|\int_{0}^{T} \int_{\Omega} \nabla^{\perp} \psi_{n} \cdot[\Lambda, \nabla \varphi] \psi_{n} d x \phi d t-\int_{0}^{T} \int_{\Omega} \nabla^{\perp} \psi \cdot[\Lambda, \nabla \varphi] \psi d x \phi d t\right| \\
& \leq\left|\int_{0}^{T} \int_{\Omega} \nabla^{\perp}\left(\psi_{n}-\psi\right) \cdot[\Lambda, \nabla \varphi] \psi d x \phi d t\right|+\left\|\phi \nabla^{\perp} \psi_{n}\right\|_{L^{2}\left(0, T ; L^{2}(\Omega)\right)}\left\|[\Lambda, \nabla \varphi]\left(\psi_{n}-\psi\right)\right\|_{L^{2}\left(0, T ; L^{2}(\Omega)\right)} \\
& \leq\left|\int_{0}^{T} \int_{\Omega} \nabla^{\perp}\left(\psi_{n}-\psi\right) \cdot[\Lambda, \nabla \varphi] \psi d x \phi d t\right|+C\left\|\psi_{n}-\psi\right\|_{L^{2}\left(0, T ; D\left(\Lambda^{\frac{1}{2}}\right)\right)} .
\end{aligned}
$$

The first term converges to 0 due to the weak convergence of $\psi_{n}$ to $\psi$ in $L^{2}\left(0, T ; H_{0}^{1}(\Omega)\right)$ and the fact that $[\Lambda, \nabla \varphi] \psi \in D\left(\Lambda^{\frac{1}{2}}\right) \subset L^{2}(\Omega)$ in view of Theorem 2.3. By interpolation, the second term is bounded by

$$
\left\|\psi_{n}-\psi\right\|_{L^{2}\left(0, T ; D\left(\Lambda^{\frac{1}{2}}\right)\right)} \leq\left\|\psi_{n}-\psi\right\|_{L^{2}\left(0, T ; L^{2}(\Omega)\right)}^{\frac{1}{2}}\left\|\psi_{n}-\psi\right\|_{L^{2}(0, T ; D(\Lambda))}^{\frac{1}{2}} \leq C\left\|\psi_{n}-\psi\right\|_{L^{2}\left(0, T ; L^{2}(\Omega)\right)}^{\frac{1}{2}}
$$

which also converge to 0 . Finally, we apply the commutator estimate in Theorem 2.5 to obtain

$$
\begin{aligned}
& \left|\int_{0}^{T} \int_{\Omega}\left[\Lambda, \nabla^{\perp}\right] \psi_{n} \cdot \nabla \varphi \psi_{n} d x \phi d t-\int_{0}^{T} \int_{\Omega}\left[\Lambda, \nabla^{\perp}\right] \psi \cdot \nabla \varphi \psi d x \phi d t\right| \\
& \leq\left\|\nabla \varphi\left[\Lambda, \nabla^{\perp}\right]\left(\psi_{n}-\psi\right)\right\|_{L^{2}\left(0, T ; L^{2}(\Omega)\right)}\left\|\phi \psi_{n}\right\|_{L^{2}\left(0, T ; L^{2}(\Omega)\right)} \\
& \quad+\left\|\left[\Lambda, \nabla^{\perp}\right] \psi \cdot \nabla \varphi\right\|_{L^{2}\left(0, T ; L^{2}(\Omega)\right)}\left\|\phi\left(\psi_{n}-\psi\right)\right\|_{L^{2}\left(0, T ; L^{2}(\Omega)\right)} \\
& \leq C\left\|\psi_{n}-\psi\right\|_{L^{2}\left(0, T ; L^{2}(\Omega)\right)}
\end{aligned}
$$

which converge to 0 . Putting together the above considerations leads to

$$
\int_{0}^{T} \int_{\Omega} \theta \varphi(x) d x \partial_{t} \phi(t) d t+\int_{0}^{T} \int_{\Omega} u \theta \cdot \nabla \varphi(x) d x \phi(t) d t=0, \quad \forall \phi \in C_{c}^{\infty}((0, T)), \varphi \in C_{c}^{\infty}(\Omega) .
$$

Therefore, $\theta$ is a weak solution of the inviscid SQG equation on $[0, T]$. Finally, consider $s \in(0,1]$. We have the the balance (1.6) for each $\theta_{n}$. Since $s \leq 1$ the uniform boundedness of $\theta_{n}$ in $L^{\infty}\left(0, T ; L^{2}(\Omega)\right)$ implies

$$
\lim _{n \rightarrow \infty} \nu_{n} \int_{0}^{t} \int_{\Omega}\left|\Lambda^{\frac{s-1}{2}} \theta_{n}\right|^{2} d x d r=0, \quad t \in[0, T] .
$$

In addition, $\theta_{n} \rightarrow \theta$ strongly in $C\left(0, T ; D\left(\Lambda^{-\varepsilon}\right)\right) \subset C\left(0, T ; D\left(\Lambda^{-\frac{1}{2}}\right)\right)$. Letting $\nu=\nu_{n} \rightarrow 0$ in the balance (1.6) we conclude that the Hamiltonian of $\theta$ is constant on $[0, T]$. 


\section{Appendix A. A bound on $\mathbb{P}_{m}$}

Recall the definition (3.1) of $\mathbb{P}_{m}$. The following lemma is essentially taken from [7]. We include the proof for the sake of completeness.

LEMMA A.1. Let $\Omega \subset \mathbb{R}^{d}, d \geq 2$, be a bounded domain with smooth boundary. For every $N$ and $k \in \mathbb{N}$ satisfying $N>\frac{k}{2}+\frac{d}{2}$ there exists a positive constant $C_{N, k}$ such that

$$
\left\|\mathbb{P}_{m} \varphi\right\|_{H^{k}(\Omega)} \leq C_{N, k}\|\varphi\|_{D\left(\Lambda^{2 N}\right)}
$$

for all $m \geq 1$ and $\phi \in D\left(\Lambda^{2 N}\right)$; moreover, we have

$$
\lim _{m \rightarrow \infty}\left\|\left(\mathbb{I}-\mathbb{P}_{m}\right) \varphi\right\|_{H^{k}(\Omega)}=0 .
$$

Proof. As $\varphi \in D\left(\Lambda^{2 N}\right)$, we have $\Delta^{\ell} \varphi \in H_{0}^{1}(\Omega)$ for all $\ell=0,1, \ldots, N-1$. This allows repeated integration by parts with $w_{j}$ using the relation $-\Delta w_{j}=\lambda_{j} w_{j}$. Using Hölder's inequality and the fact that $w_{j}$ is normalized in $L^{2}$, we obtain

$$
\left|\varphi_{j}\right| \leq \lambda_{j}^{-N}\left\|\Delta^{N} \varphi\right\|_{L^{2}}, \quad \varphi_{j}=\int_{\Omega} \varphi w_{j} d x .
$$

By elliptic regularity estimates and induction, we have for all $k \in \mathbb{N}$ that

$$
\left\|w_{j}\right\|_{H^{k}(\Omega)} \leq C_{k} \lambda_{j}^{\frac{k}{2}}
$$

We know from the easy part of Weyl's asymptotic law that $\lambda_{j} \geq C j^{\frac{2}{d}}$. Consequently, with $N>\frac{k}{2}+\frac{d}{2}$ we deduce that

$$
\begin{aligned}
\sum_{j=1}^{\infty}\left|\varphi_{j}\right|\left\|w_{j}\right\|_{H^{k}(\Omega)} & \leq C_{k}\left\|\Delta^{N} \varphi\right\|_{L^{2}} \sum_{j=1}^{\infty} \lambda_{j}^{-N+\frac{k}{2}} \\
& \leq C_{k}\|\varphi\|_{D\left(\Lambda^{2 N}\right)} \sum_{j=1}^{\infty} j^{\left(-N+\frac{k}{2}\right) \frac{2}{d}} \\
& =C_{N, k}\|\varphi\|_{D\left(\Lambda^{2 N}\right)}
\end{aligned}
$$

where $C_{N, k}<\infty$ depends only on $N$ and $k$. Because

$$
\left(\mathbb{I}-\mathbb{P}_{m}\right) \varphi=\sum_{j=m+1}^{\infty} \varphi_{j} w_{j},
$$

this proves both A.1 and A.2. The proof is complete.

Acknowledgment. The research of PC was partially supported by NSF grant DMS-1713985.

\section{References}

[1] L. C. Berselli. Vanishing Viscosity Limit and Long-time Behavior for 2D Quasi-geostrophic Equations. Indiana Univ. Math. J. 51(4) (2002), 905-930.

[2] T. Buckmaster, S. Shkoller, V. Vicol. Nonuniqueness of weak solutions to the SQG equation. arXiv:1610.00676, to appear in Communications on Pure and Applied Mathematics.

[3] D. Chae, P. Constantin, D. Córdoba, F. Gancedo, J. Wu. Generalized surface quasi-geostrophic equations with singular velocities. Comm. Pure Appl. Math., 65 (2012) No. 8, 1037-1066.

[4] P. Constantin, D. Cordoba, J. Wu. On the critical dissipative quasi-geostrophic equation. Indiana Univ. Math. J., 50 (Special Issue): 97-107, 2001. Dedicated to Professors Ciprian Foias and Roger Temam (Bloomington, IN, 2000).

[5] P. Constantin, M. Ignatova. Remarks on the fractional Laplacian with Dirichlet boundary conditions and applications. Internat. Math. Res. Notices, (2016), 1-21. 
[6] P. Constantin, M. Ignatova. Critical SQG in bounded domains. Ann. PDE (2016) 2:8.

[7] P. Constantin, H.Q. Nguyen. Global weak solutions for SQG in bounded domains. arXiv:1612.02489, to appear in Comm. Pure Appl. Math.

[8] P. Constantin, H. Q. Nguyen. Local and global strong solutions for SQG in bounded domains. arXiv:1705.05342, to appear in Physica D, Special Issue in Honor of Edriss Titi.

[9] P. Constantin, A.J. Majda, and E. Tabak. Formation of strong fronts in the 2-D quasigeostrophic thermal active scalar. Nonlinearity, 7(6) (1994), 1495-1533.

[10] P. Constantin, A. Tarfulea, V. Vicol. Absence of anomalous dissipation of energy in forced two dimensional fluid equations. Arch. Ration. Mech. Anal. 212 (2014), 875-903.

[11] F. Marchand. Existence and Regularity of Weak Solutions to the Quasi-Geostrophic Equations in the Spaces $L^{p}$ or $\dot{H}^{-1 / 2}$. Comm. Math. Phys. (2008) 277(1): 45-67.

[12] I.M. Held, R.T. Pierrehumbert, S.T. Garner, and K.L. Swanson. Surface quasi-geostrophic dynamics. J. Fluid Mech., 282 (1995),1-20.

[13] P. Isset and V. Vicol. Hölder continuous solutions of active scalar equations. Ann. PDE 1 (2015), no. 1, 1-77.

[14] H. Q. Nguyen. Global weak solutions for generalized SQG in bounded domains. Anal. PDE, Vol. 11 (2018), No. 4, 1029-1047.

[15] J.L. Lions, Quelque methodes de résolution des problemes aux limites non linéaires. Paris: Dunod-Gauth, 1969.

[16] J. L. Lions, E. Magenes, Non-homogeneous boundary value problems and applications. Vol. I. Translated from the French by P. Kenneth. Die Grundlehren der mathematischen Wissenschaften, Band 181. Springer-Verlag, New York-Heidelberg, 1972.

[17] S. Resnick, Dynamical problems in nonlinear advective partial differential equations. ProQuest LLC, Ann Arbor, MI, 1995, Thesis (Ph.D.)-The University of Chicago.

Department of Mathematics, Princeton University, Princeton, NJ 08544

E-mail address: const@math.princeton.edu

Department of Mathematics, Princeton University, Princeton, NJ 08544

E-mail address: ignatova@math.princeton.edu

Department of Mathematics, Princeton University, Princeton, NJ 08544

E-mail address: qnemath.princeton.edu 